\title{
A Study on the High-efficiency Coreless-typed Wireless Power Transfer System for Railway Transit
}

\begin{abstract}
Chan-Bae Park ${ }^{\dagger}$
Abstract - This paper presents the derivation and investigation of a practical model structure for a large-capacity WPT system capable of powering a railway system. Comparative analyses of the secondary pickup induced voltages and rail induced voltages for various WPT models are conducted to derivate the optimal WPT model which could be applied to railway vehicles. Through the analyses for various WPT models, a coreless-type WPT model structure, which has the same dimension and performance as the secondary pickup module of the conventional core-type WPT model, is derived. In order to verify the characteristic analysis results from the simulation method of the core-type and coreless-type WPT models, small-scaled proto-models are manufactured for both the conventional core-type WPT model and the coreless-type model which has the best performance. It is confirmed that the coreless-type WPT model could have similar performance with the core-type WPT model through performance tests.
\end{abstract}

Keywords: Wireless power transfer, WPT, Core-type, Coreless-type, Ground power feeding line

\section{Introduction}

A Wireless Power Transfer (WPT) system is a contactless power feeding device from a source to a load. Its application scope ranges from small-capacity systems such as electronic toothbrush chargers and cell phone chargers to large-capacity systems such as electric vehicles and industrial transport equipment [1,2]. Different from the conventional catenary/pantograph power feeding methods, WPT systems are being currently introduced as a new mechanism for energy transfer in railway systems and various research studies are being conducted related to the application of WPT systems for trains [3-6]. Bombardier developed a three-phase $200 \mathrm{~kW} / 20 \mathrm{kHz}$-class WPT system and demonstrated to mount it on a PRIMOVE tram in 2008 [7]. However, Bombardier's WPT system was not suitable for the simultaneous power transfer of multiple pickups because the three-phase variable magnetic flux travels along the ground power feeding line. Some research shows that a single-phase WPT system is more advantageous to deliver power simultaneously to multiple pickups [4]. In 2013, Korea Railroad Research Institute (KRRI) and Korea Advanced Institute of Science and Technology (KAIST) proposed a ground power feeding line and pickup designs for a single-phase $180 \mathrm{~kW} / 60 \mathrm{kHz}$-class WPT system and demonstrated to mount it on a Wireless-Tram. Fig. 1 shows the $180 \mathrm{~kW} / 60 \mathrm{kHz}$-class WPT system applied to a Wireless-Tram. Many researches up until now have been focused on ensuring performance not reducing size and

$\dagger$ Corresponding Author: Dept. of Railroad Operation System Engineering, Korea National University of Transportation, Korea. (cbpark@ut.ac.kr)

Received: March 3, 2016; Accepted: May 5, 2016 cost. However, the output power of the WPT system, about $10 \mathrm{MW}$ is required in order to apply a WPT system to a large-capacity system such as a high-speed railway vehicle. The 10MW-class WPT system is a difficult task in size and cost. Ultimately, a variety of technologies for size and cost reduction as well as energy transfer performance are critically needed to secure the technology for the largecapacity WPT system $[8,9]$.

In general, a loosely coupled WPT system is well-known to be suitable as a high-efficiency, high-power, large airgap system. Therefore, the topology is selected as an alternative to the conventional contacted power feeding system. However, the magnetic material is used excessively on the conventional WPT model. Therefore, the purpose of this research is to investigate a practical model for the large-capacity WPT system capable of powering a railway system. Therefore, the derivation of the optimal WPT model, which could be applied to either urban railway vehicles or high-speed railway vehicles, is conducted in this study. This paper is structured as follows. A simulation approach is used to analyze the characteristics for structure

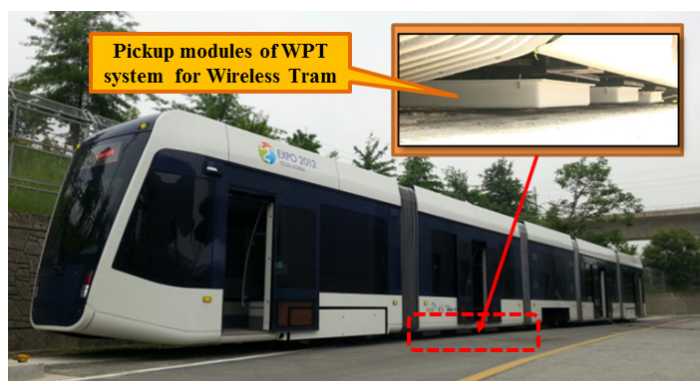

Fig. 1. $180 \mathrm{~kW} / 60 \mathrm{kHz}$-class WPT system applied to Wireless-Tram 
topology of the ground coil and on-board pick-ups on a coreless-type WPT model. The characteristic variations of the equivalent circuit parameters and the coupling coefficient $\mathrm{k}$ on each coreless-type WPT model are compared. Subsequently, in order to verify the characteristic analysis results from the simulation method of the coreless-type WPT model, two small-scaled proto-models are manufactured and tested for both the conventional core-type WPT model and the coreless-type model.

\section{Design of the Coreless-type WPT Model}

\subsection{Configuration of a WPT system for Wireless- tram}

Fig. 2 shows an electric block diagram comparison between the conventional tram and the Wireless-tram. An electric block diagram for a conventional tram is shown in Fig. 2 (a). $750 \mathrm{Vdc}$ power received from the catenary and pantograph is transferred to the battery through the battery charger in charging mode. In the traveling mode, the power stored in the battery is supplied to the traction inverter. An electric block diagram for Wireless-tram using WPT system is shown in Fig. 2 (b). $60 \mathrm{kHz}$-frequency resonant inverter, which is installed on the way side, transfers AC power to the series-tuned ground power feeding line. Then, the ground power feeding coil generates high-frequency magnetic fields that couple with the pickup coils installed at the bottom of the Wireless-tram. The pickups transfer the power to the voltage regulator. Finally, the voltage regulator supplies a constant voltage to the battery charger.

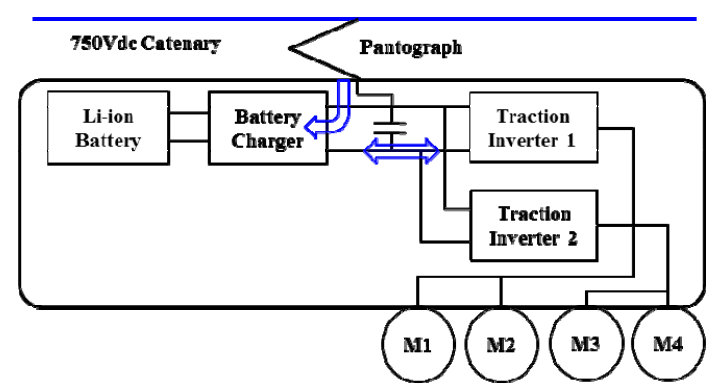

(a) Conventional tram

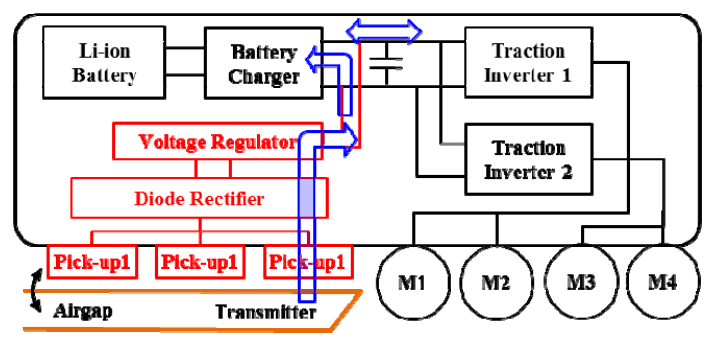

(b) Wireless-tram

Fig. 2. Electric block diagram comparison between the conventional tram and the Wireless-tram
Consequently, the WPT system replaces the pantograph / catenary system.

\subsection{The conventional core-type WPT model}

Fig. 3 shows the conventional $60 \mathrm{~kW}$-class WPT model and ground power feeding line applied to the WirelessTram. As shown in Fig. 3, the ground power feeding line has a lot of ferrite-core. In this core-type WPT model, the construction for the ground power feeding line and equipment is difficult and the cost is high because the ground power feeding system has to be installed throughout the entire tram route. Therefore, derivation of a coreless-type WPT model with low-cost structure is necessary. In general, the flux linkage into the secondary pickup coil is reduced when the ferrite core is eliminated from the ground power feeding line. Consequently, in order to apply a coreless-type ground power feeding line to WPT model, a derivation of an appropriate secondary pickup structure is necessary. Table 1 shows specifications for the conventional 60kW-class WPT model.

\subsection{Structure design and property analysis of the coreless-type WPT model}

In general, a loosely coupled WPT system is well-known to be suitable as a high-efficiency, high-power and large air-gap system. Therefore the loosely coupled topology is suitable as WPT system for a train [10]. The loosely coupled WPT system is less sensitive to changes in the

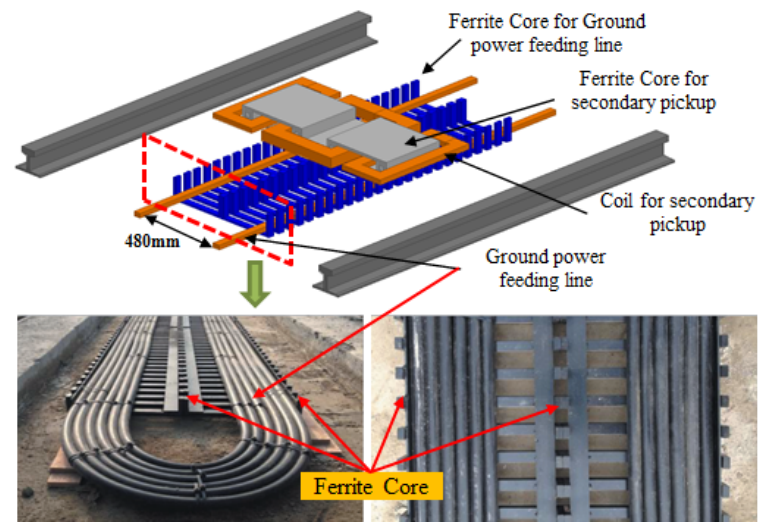

Fig. 3. $60 \mathrm{~kW} / 60 \mathrm{kHz}$-class WPT model and ground power feeding line applied to Wireless-Tram

Table 1. Specifications for the conventional $60 \mathrm{~kW}$-class core-type WPT model

\begin{tabular}{c|c}
\hline Contents & Value \\
\hline Output power per module & $60 \mathrm{~kW}$ \\
\hline Resonant frequency & $60 \mathrm{kHz}$ \\
\hline Pickup module size & $725 * 270 * 87 \mathrm{~mm}$ \\
\hline Air gap & $70 \mathrm{~mm}$ \\
\hline MMF of ground coil & $240 * 4$ Aturns \\
\hline Pickup turns & $200(4 \mathrm{CH})$ Turns \\
\hline
\end{tabular}




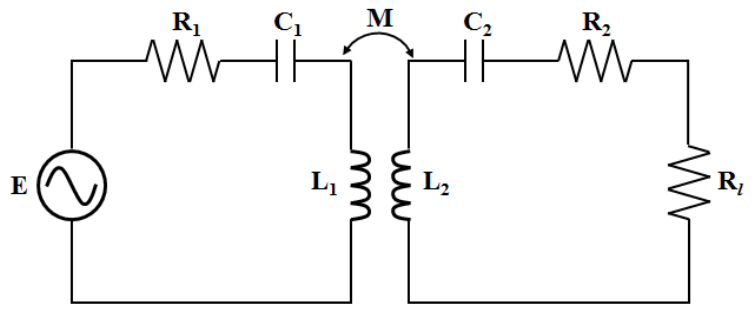

Fig. 4. Equivalent circuit of the series-series WPT model

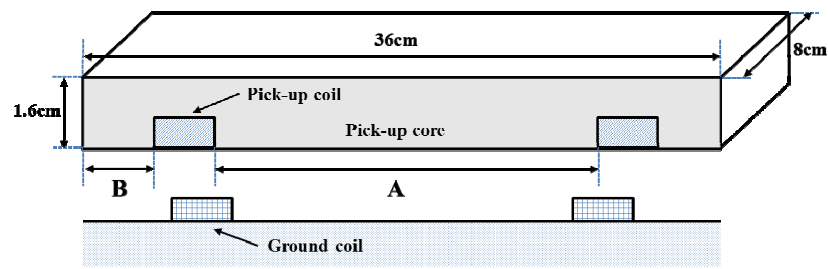

Fig. 5. Structure and design parameters of the secondary pickup module for the coreless-type WPT model

dimension of the WPT model. In other words, the characteristic parameters variation of the loosely coupled WPT system is less sensitive to changes in the shape and dimension. Therefore, the coreless-type WPT model, which is derived from the optimal design process, is available as an alternative to the conventional WPT model.

The equivalent circuit of the series-series resonant system, which is applied to the conventional core-type WPT system, is shown in Fig. 4. $R_{1}$ and $R_{2}$ are equivalent series resistance of the ground power feeding line and pickup coils. $C_{1}$ and $C_{2}$ are compensating capacitors and $R_{l}$ is load resistance. $a$ is the transfer ratio and $w_{o}$ is the resonant frequency. In general, the power transfer efficiency $\eta_{s s}$ of the magnetic resonant system is represented by the Eq. (1) [11]. As shown in the Eq. (1) and (2), the power transfer efficiency $\eta_{s s}$ is proportional to the square of the coupling coefficient $k$ and is sensitive to changes in the coupling coefficient $k$. Therefore, the design of a corelesstype WPT model with the large coupling coefficient $\mathrm{k}$ is conducted in this study.

$$
\begin{gathered}
\eta_{s s}=1 /\left\{1+\frac{R_{2}}{R_{l}}+\left(\frac{R_{l}+R_{2}}{a \cdot w_{0} \cdot L_{2}}\right)^{2} \cdot \frac{R_{1}}{R_{l}}\right\} \\
a=k \sqrt{L_{1} / L_{2}}
\end{gathered}
$$

In order to apply a coreless-type ground power feeding line to WPT model, a new design of the appropriate secondary pickup module structure is necessary. Above all, a simple plate-shaped ferrite core structure is applied to the secondary pickup module for low-cost manufacturing. In this paper, a small-scaled coreless-type WPT model is designed for performance comparison with the core-type WPT model. Fig. 5 shows the structure and design parameters of the secondary pickup module for the small-
Table 2. Levels of the design variables on the secondary pickup module

\begin{tabular}{c|c|c|c|c}
\hline Variables & Model 1 & Model 2 & Model 3 & Model 4 \\
\hline $\mathrm{A}$ & $25.7 \mathrm{~cm}$ & $21.3 \mathrm{~cm}$ & $16.9 \mathrm{~cm}$ & $12.5 \mathrm{~cm}$ \\
\hline $\mathrm{B}$ & $0 \mathrm{~cm}$ & $2.2 \mathrm{~cm}$ & $4.4 \mathrm{~cm}$ & $6.6 \mathrm{~cm}$ \\
\hline
\end{tabular}

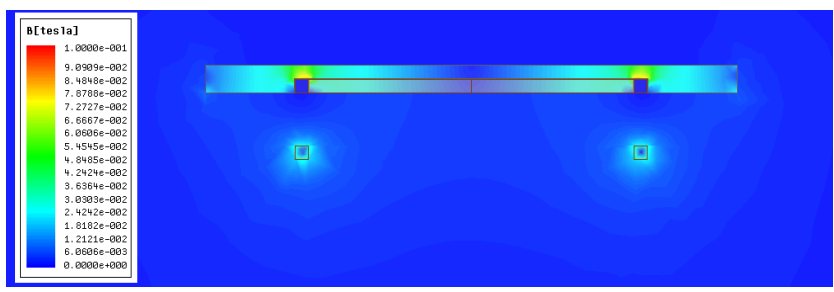

Fig. 6. Magnetic flux density distributions of the derived coreless-type WPT model under no-load conditions

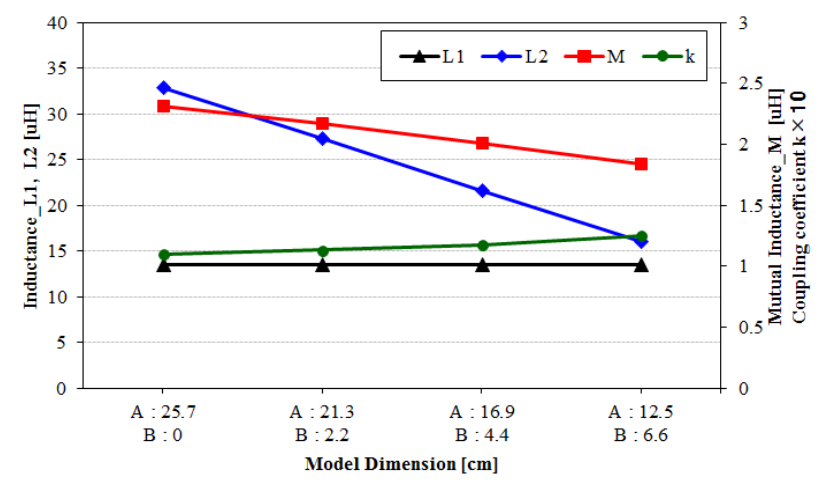

Fig. 7. Characteristic variations of the equivalent circuit parameters and the coupling coefficient $k$ on each coreless-type WPT model

scaled coreless-type WPT model design. As shown in Fig. 5 , the secondary pickup module for the coreless-type WPT model has 2 design variables. ' $A$ ' is the ferrite core width inside the pickup coils and ' $\mathrm{B}$ ' is the ferrite core width outside the pickup coils. Table 2 shows the levels of the design variables on the secondary pickup module. Fig. 6 shows the magnetic flux density distributions of the derived coreless-type WPT model under no-load conditions. As shown in Fig. 6, the magnetic flux density at the secondary pickup core is below $0.1 \mathrm{~T}$. Thus, there seem to be no issues with saturation during the rated operations. Fig. 7 shows the characteristic variations of the equivalent circuit parameters and the coupling coefficient $k$ on each coreless-type WPT model according to a change of the design variables. As shown in Fig. 6, the coupling coefficient $k$ increases as the width 'A' decreases. Fig. 8 shows the characteristic variations of the power transfer efficiency $\eta_{s s}$ according to a change of the load resistance. As shown in Fig. 8, the power transfer efficiency $\eta_{s s}$ of the Model 4(A: $12.5 \mathrm{~cm}, \mathrm{~B}: 6.6 \mathrm{~cm})$ is the largest. In addition, the efficiency difference of each model is large when the load resistance is small, but the difference decreases as the load resistance 
increases. Based on the above results, the Model 4(A: $12.5 \mathrm{~cm}, \mathrm{~B}: 6.6 \mathrm{~cm})$ is selected as the secondary pickup module of the small-scaled coreless-type WPT model.

\section{Production of Small-scaled WPT Models and Verification by Performance test}

\subsection{Production of small-scaled WPT proto-models}

In this study, 2 small-scaled proto-models are manufactured to verify the characteristic analysis results from the simulation method between the core-type and coreless-type WPT model. First, a $6 \mathrm{KW}$-class PAM(Pulse Amplitude Modulation) resonant inverter is created to supply high-frequency power to the WPT models. Next, small-scaled WPT proto-models are created for both the conventional core-type WPT model and the coreless-type WPT model. The ground power feeding lines for each small-scaled WPT proto-model have 4 turns and the secondary pickup modules have 8 turns. The specifications for each small-scaled WPT proto-model are provided in Table 3. The inductance and capacitance parameters of the ground power feeding lines and the secondary pickup

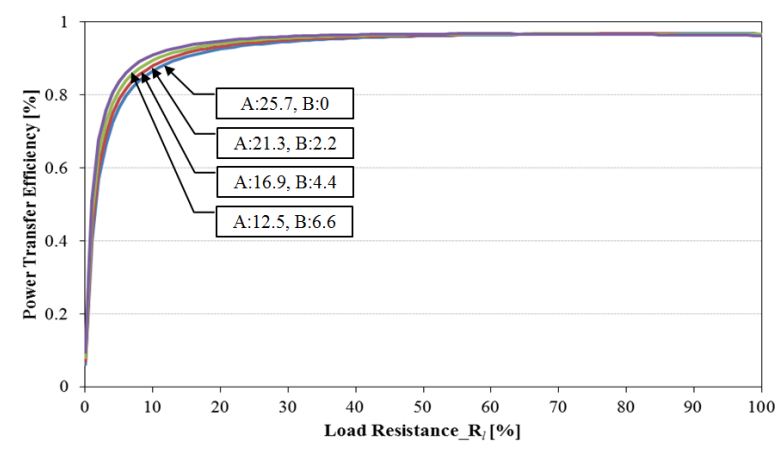

Fig. 8. Characteristic variations of the power transfer efficiency on each coreless-type WPT model

Table 3. Specifications for the completed small-scaled WPT proto-models

\begin{tabular}{c|c|c}
\hline Contents & Core-Type & Coreless-Type \\
\hline Width of GC & $13.8 \mathrm{~cm}$ & $17.5 \mathrm{~cm}$ \\
\hline Turns of GC/ PC & $4 / 8$ & $4 / 8$ \\
\hline $\mathrm{R}_{1} / \mathrm{L}_{1} / \mathrm{C}_{1}$ of GC & $0.0155 \Omega / 20.7 \mathrm{uH} /$ & $0.0159 \Omega / 14.8 \mathrm{uH} /$ \\
& $0.33 \mathrm{uF}$ & $0.46 \mathrm{uF}$ \\
\hline Size of PC & $32.3 * 9.5 * 3.2 \mathrm{~cm}$ & $35.7 * 8 * 1.6 \mathrm{~cm}$ \\
\hline $\mathrm{R}_{2} / \mathrm{L}_{2} / \mathrm{C}_{2}$ of PC & $0.0124 \Omega / 14.8 \mathrm{uH} /$ & $0.0106 \Omega / 17.3 \mathrm{uH} /$ \\
& $0.44 \mathrm{uF}$ & $0.39 \mathrm{uF}$ \\
\hline \multicolumn{2}{|r}{}
\end{tabular}

Table 4. Performance indexes for the completed smallscaled WPT proto-models

\begin{tabular}{c|c|c}
\hline Contents & Core-Type & Coreless-Type \\
\hline Resonant Frequency & $61 \mathrm{kHz}$ & $61 \mathrm{kHz}$ \\
\hline Air gap g & $35 \mathrm{~mm}$ & $35 \mathrm{~mm}$ \\
\hline Mutual Inductance M & $2.46 \mathrm{uH}$ & $2.53 \mathrm{uH}$ \\
\hline Coupling Coefficient $\mathrm{k}$ & 0.138 & 0.158 \\
\hline
\end{tabular}

modules are included in Table 3. Table 4 shows performance indexes for the completed small-scaled WPT proto-models. As shown in Table 4, the coreless-type WPT model is designed to be larger than the core-type WPT model in the mutual inductance and coupling coefficient $\mathrm{k}$. Fig. 9 shows the completed small-scaled WPT protomodels.

\subsection{Verification through performance test of the small-scaled WPT proto-models}

In this study, load and no-load tests are conducted for both of the manufactured small-scaled WPT system protomodels. Fig. 10 shows the arrangement diagram of the small-scaled WPT system for performance test. The operating frequency and the output current of the resonant inverter are approximately $64 \mathrm{kHz}, 60 \mathrm{~A}$, respectively. Bifurcation gaping resonance occurs when the ground power feeding line and the pick-up coil having the same resonant frequency is located close to each other. In this case, if the operating frequency of the resonant inverter is $61 \mathrm{kHz}$, a capacitive component in the output of the resonant inverter is shown and there is a risk of burn out in the resonant inverter. Therefore, the operating frequency of the resonant inverter is set at $64 \mathrm{kHz}$ higher than the resonant frequency $(61 \mathrm{kHz})$ of the ground power feeding line. Fig. 11 shows the measurement results of the no-load induced voltage tests for each of the WPT proto-models. Fig. 11 (a) and (b) show the measured wave patterns of the induced voltage at $35 \mathrm{~mm}$ air gap, and Fig. 11 (c) shows the variation properties of the induced voltages according to

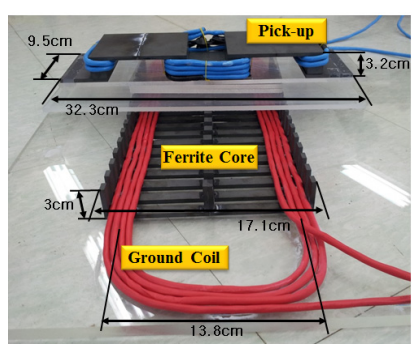

(a) Core-type

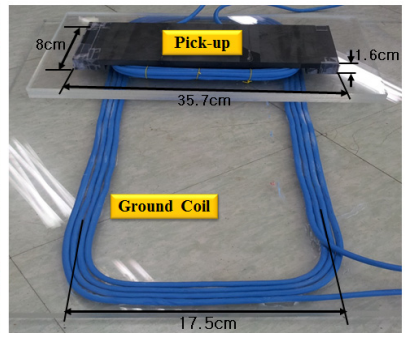

(b) Coreless-type
Fig. 9. Completed small-scaled proto-WPT models

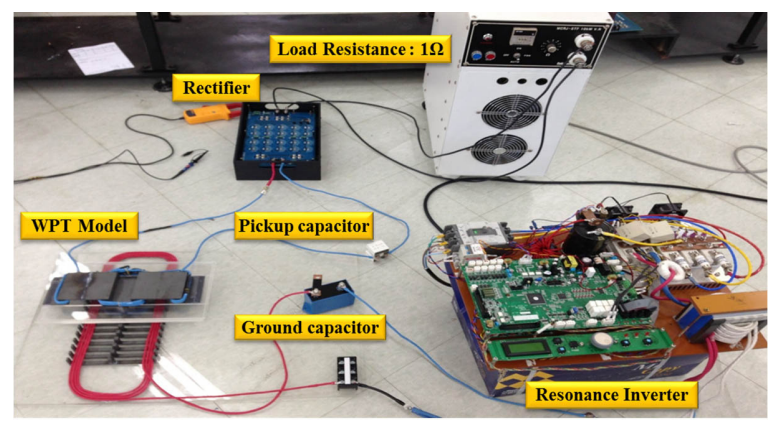

Fig. 10. Arrangement diagram for performance test 


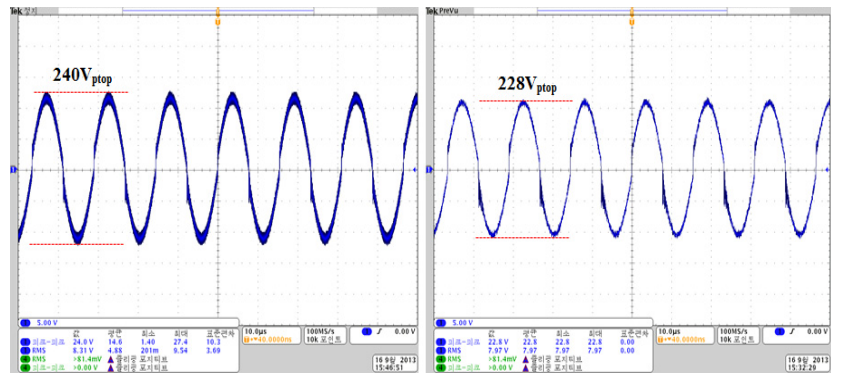

(a) Core-type $@ g=35 \mathrm{~mm}$

(b) Coreless-type $@ g=35 \mathrm{~mm}$

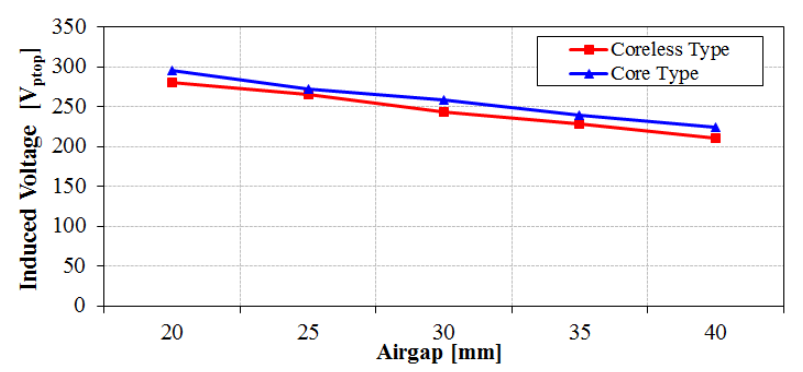

(c) Properties of the induced voltages

Fig. 11. Measurement results of the no-load induced voltage tests

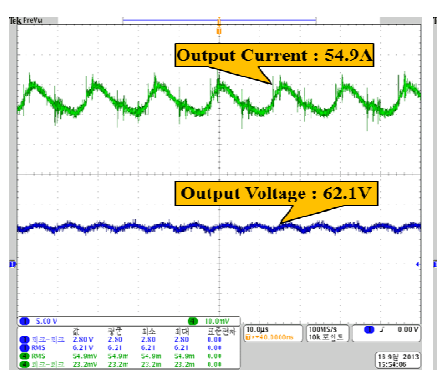

(a) Core-type@g=35mm

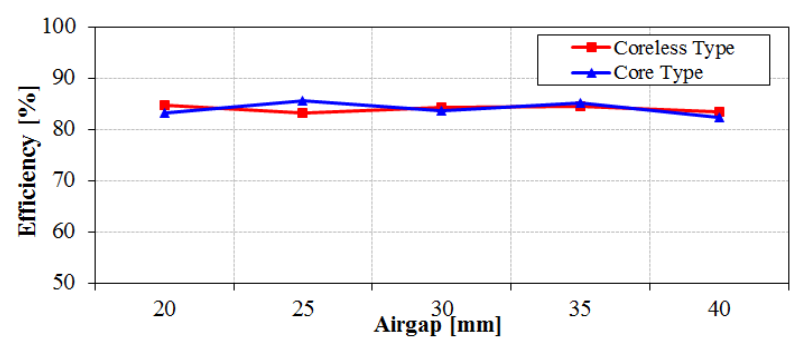

(c) Properties of the induced voltages

Fig. 12. Measurement results of the rectifier output voltage / current, and system efficiency under load conditions

variation of air gap. As shown in Fig. 11, the no-load induced voltage for the coreless-type WPT proto-model is approximately 5\% smaller than that of the core-type WPT proto-model. Fig. 12 shows the measurement results of the rectifier output voltage/current, and system efficiency according to differences of air gap for each of the WPT proto-models under load conditions. A load resistor is $1 \Omega$. The output power of both small-scaled WPT proto-models is maintained at $3.18 \mathrm{~kW}$. Fig. 12 (a) and (b) show the wave patterns of the voltage and current measured at the rectifier output terminals for each WPT proto-model at $35 \mathrm{~mm}$ air gap. Fig. 12 (c) shows the measurement results of the overall system efficiency according to differences of air gap for each of the WPT proto-models under load conditions. In this study, the efficiency of the WPT system is derived by measuring the following: the DC-link voltage of the resonant inverter's input terminal and the output voltage of the secondary pickup module's rectifier output terminal. As shown in Fig. 12 (c), when the output power of the secondary pickup module's rectifier is $3.18 \mathrm{~kW}$, the efficiency of both the core-type WPT proto-model and the coreless-type WPT proto-model is approximately $75 \%$. Additionally, even if the air gap is changed from $20 \mathrm{~mm}$ to $40 \mathrm{~mm}$, there is almost no change in efficiency. If the resonant inverter is operated at the resonant frequency of the WPT system, the efficiency is about $95 \%$ at load resistance of $1 \Omega$ in theory, but the experimental results are about $84 \%$. In conclusion, the performance test confirms that the coreless-type WPT model could have similar performance with the core-type WPT model by proper design in the loosely coupled WPT system. Therefore, it is predicted that the developed WPT system will be able to meet the magnetic-field radiation and induced magneticfield safety guidelines.

\section{Conclusion}

The derivation and investigation of a practical model structure for the large-capacity WPT system capable of powering a railway system are conducted in this paper. The derivation of the optimal coreless-type WPT model applied to either urban railway vehicles or high-speed railway vehicles is conducted in this study. The characteristic variations of the equivalent circuit parameters and the coupling coefficient $\mathrm{k}$ on each coreless-type WPT model are compared. In order to verify the characteristic analysis results from the simulation method of the coreless-type WPT model, two small-scaled proto-models are manufactured and tested for both the conventional core-type WPT model and the coreless-type model in order to determine which has the best performance. It is confirmed that the coreless-type WPT model could have similar performance with the core-type WPT model through the performance tests. In this study, the ground power feeding line without the ferrite core and the simplified structure of the secondary pick-up module for the coreless-type WPT model are derived. It is expected that the manufacturing and durability of the product will be improved and production costs will be reduced when the WPT module is applied to large-capacity systems for high-speed railways in the future. 


\section{Acknowledgements}

This research was supported by Korea National University of Transportation, Republic of Korea in 2016.

\section{References}

[1] M. Budhia, G. A. Covic, J. T. Boys, "Design and optimization of magnetic structures for lumped inductive power transfer systems," Energy Conversion Congress and Exposition 2009(ECCE2009), pp. 20812088, 2009.

[2] C. S. Wang, G. A. Covic, and O. H. Stielau, "Power Transfer Capability and Bifurcation Phenomena of Loosely Coupled Inductive Power Transfer System," IEEE Transactions on Industrial Electronics, Vol. 51, pp. 148-157, 2004.

[3] A. Kawamura, G. Kuroda, and C. Zhu, "Experimental Results on Contact-less Power Transmission System for the High-speed Trains," Power Electronics Specialists Conference 2007, pp. 2779-2784, 2007.

[4] J. Winter, S. Mayer, and S. Kaimer, "Inductive Power Supply For Heavy Rail Vehicles," 3rd International Electric Drives Production Conference 2013, 2013.

[5] S. Y. R. Hui and W. C. Ho, "A new generation of universal contactless battery charging platform for portable consumer electronic equipment," IEEE Transactions on Power Electronics, Vol. 20, No. 3 pp. 620-627, 2005.

[6] K. Hwang, S. Kim, S. Kim, Y. Chun, and S. Ahn, "Design of Wireless Power Transfer System for Railway Application," International Journal of Railway, Vol. 5, No. 4, pp. 167-174, 2012.

[7] K. Woronowicz, "System and method for transferring electric energy to a vehicle using segments of a conductor arrangement which can be operated independently," May 2014, US Patent App. 14/124, 301.

[8] Z. N. Low, R. A. Chinga, R. Tseng, and J. Lin, "Design and Test of a High-Power High-efficiency Loosely Coupled Planar Wireless Power Transfer System," IEEE Transactions on Industrial Electronics, Vol.56, pp. 1801-1812, 2009.

[9] K. Fotopoulou, B. W. Flynn, "Wireless Power Transfer in Loosely Coupled Links: Coil Misalignment Model," IEEE Transactions on Magnetics, Vol. 47, pp. 416-430, 2011.

[10] S. H. Lee, and R. D. Lorenz, "Development and Validation of Model for 95\%-Efficiency 220-W Wireless Power Transfer over a 30-cm Air gap," IEEE Transactions on Industry Applications, Vol. 47, pp. 2495-2504, 2011.

[11] Awai I., "New expression for coupling coefficient between resonators," IECE Trans., Electron, Vol. E88-3, No. 12, pp. 2295-2301, 2005.

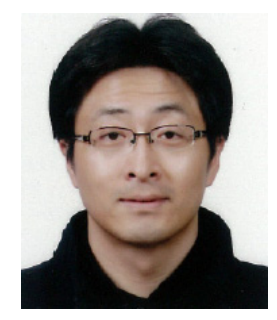

Chan-Bae Park He received the M.S. degree in electrical engineering from Seoul National University, Seoul, Korea, in 2003 and the Ph.D. degree in electrical engineering from Hanyang University, Seoul, Korea, in 2013. From 2003 to 2006, he had worked as Senior Engineer in the Digital Appliance R\&D Center at Samsung Electronics. From 2007 to 2015, he had worked as Senior Researcher at the Korea Railroad Research Institute. Since 2015, he has been an Assistant Professor at the Korea National University of Transportation, Uiwangsi, Korea. His research interests include design and analysis of motor/generator, transformer, and superconducting devices for energy conversion systems. 